\title{
Nonlinear Periodic and Solitary Water Waves on Currents in Shallow Water
}

\author{
By Roger Grimshaw and Zihua Liu
}

\begin{abstract}
A variable-coefficient Korteweg-de Vries equation is used to model the deformation of nonlinear periodic and solitary water waves propagating on a unidirectional background current, which is either flowing in the same direction as the waves, or is opposing them. As well as the usual form of the Korteweg-de Vries equation, an additional term is needed when the background current has vertical shear. This term, which has hitherto been often neglected in the literature, is linear in the wave amplitude and represents possible nonconservation of wave action. An additional feature is that horizontal shear in the background current is inevitably accompanied by a change in total fluid depth, to conserve mass, and this change in depth is a major factor in the deformation of the waves. Using a combination of asymptotic analyses and numerical simulations, it is found that waves grow on both advancing and opposing currents, but the growth is greater when the current is opposing.
\end{abstract}

\section{Variable-coefficient Korteweg-de Vries equation}

It is well-known that deep water waves propagating into a unidirectional current of increasing strength, are diminished in amplitude when the current is the same direction as the waves, but grow in amplitude when the current is in the opposite direction, see for instance the text by Phillips [1], the review article by Peregrine [2] and the literature on rogue waves, for instance,

\footnotetext{
Address for correspondence: Roger Grimshaw, Department of Mathematics, University College London, London, WC1E 6BT, UK; e-mail: r.grimshaw@ucl.ac.uk 
the early work by Smith [3] and the recent book by Kharif et al. [4]. The simplest explanation for slowly varying linear waves is through conservation of waves and wave action. Thus the linear dispersion relation for deep water waves with wavenumber $k>0$ and frequency $\omega_{0}>0$ propagating in the positive $x$-direction on a current $U$ is $\omega_{0}=U k+\omega^{*}, \omega^{*}=\sqrt{g k}$ and we suppose that $U=0$ for $x \leq 0$ where $k=k_{0}$. When $U$ varies slowly with $x$, with $|U|$ increasing in the positive $x$-direction, then $\omega_{0}$ is a constant, and so this determines the consequent variation of $k$. For an advancing current $U>0$, this implies that $k$ decreases and the group velocity $c_{g}=U+\omega_{k}^{*}$ increases, but for an opposing current $U<0, k$ increases and the group velocity decreases. The wave amplitude $a$ is then determined from the conservation of wave action flux, $c_{g}|a|^{2} / \omega^{*}$ is constant. Thus in advancing current $|a|$ decreases, but on an opposing current $|a|$ increases, and indeed becomes infinite at the stopping velocity where $c_{g}=0$, or $U=-c_{0} / 4$ where $c_{0}=\omega_{0} / k_{0}$ is the phase velocity at $x=0$, and this linear theory fails.

In this work, we examine the analogous situation for shallow water waves with a focus on oceanic applications. Although conservation of wave action again plays an essential role, there are two major differences. First in the long wave limit where dispersion is small it is necessary to take account of nonlinearity a priori. This implies that an appropriate model is a Korteweg-de Vries (KdV) equation. Second, in shallow water, it is necessary to take account of the total depth changes associated with background current variations. The relevant variable-coefficient Korteweg-de Vries $(\mathrm{vKdV})$ equation has been derived in a general setting for both surface and internal waves, see Grimshaw [5], Zhou and Grimshaw [6],

$$
\eta_{t}+c \eta_{x}+\frac{c Q_{x}}{2 Q} \eta+\mu \eta \eta_{x}+\delta \eta_{x x x}+\sigma \eta=0
$$

Here, $\eta(x, t)$ is the amplitude of the wave, and $x, t$ are space and time variables, respectively, $c(x)$ is the relevant linear long wave speed, and $Q(x)$ is the linear magnification factor, defined so that $Q \eta^{2}$ is the wave action flux density for linear long waves. The coefficients $c, Q, \mu, \delta$ are determined by the waveguide properties of the specific physical system being considered, and they are slowly varying functions of $x$. The final term $\sigma \eta$ represents nonconservative effects arising from dissipative or forcing terms in the underlying basic state, see Grimshaw [7] for s general discussion of how this term arises when considering issues about wave action conservation. The derivation of (1) assumes the usual $\mathrm{KdV}$ balance that the nonlinear term has the same order as the dispersion, that is $\eta \eta_{x}$ has the same order of magnitude as $\eta_{x x x}$, and in addition assumes that the waveguide properties, that is, the coefficients $c, Q, \mu, \delta$, vary slowly so that, for instance, $\eta Q_{x} / Q$ and $\sigma Q$ are of the same order of magnitude as the nonlinear and dispersive terms. In this scenario, the first two terms in (1) are the dominant terms, and 
hence we make the transformation

$$
A=\sqrt{Q} \eta, \quad T=\int_{0}^{x} \frac{d x}{c}, \quad X=T-t .
$$

Substitution into (1) yields, to the same order of approximation as in the derivation of (1),

$$
\begin{gathered}
A_{T}+v A A_{X}+\lambda A_{X X X}+\sigma A=0, \\
v=\frac{\mu}{c \sqrt{Q}}, \quad \lambda=\frac{\delta}{c^{3}} .
\end{gathered}
$$

The coefficients $v, \lambda$ are functions of $T$ alone. Note that although $T$ is a variable along the spatial path of the wave, we subsequently refer to it as the "time," noting that it has the dimensions of time. Similarly, although $X$ is a temporal variable (in a reference frame moving with speed $c$ ), we subsequently refer to it as the "space" variable. Finally, since we can assume $\lambda>0$ in practice, it is useful to make a further transformation yielding the canonical form

$$
\begin{gathered}
A_{\tau}+\alpha A A_{X}+A_{X X X}+\beta A=0 \\
\text { where } \tau=\int_{0}^{T} \lambda d T=\int_{0}^{x} \frac{\delta}{c^{4}} d x, \quad \alpha=\frac{v}{\lambda}, \quad \beta=\frac{\sigma}{\lambda} .
\end{gathered}
$$

We call Eq. (5) the $\mathrm{vKdV}$ equation. For the subsequent numerical simulations, a further transformation is useful

$$
A=E B, \quad B_{\tau}+\alpha E B B_{X}+B_{X X X}=0, \quad E=\exp \left(-\int_{0}^{T} \beta d T\right) .
$$

Then, Eq. (7) is solved numerically using a pseudo-spectral code and exponential time differencing with a fourth-order Runge-Kutta scheme, see [8] for some further details.

The vKdV equation (5) has two conservation laws

$$
\begin{aligned}
& \frac{\partial M}{\partial \tau}=-\beta M, \quad M=\int_{-\infty}^{\infty} A d X, \\
& \frac{\partial P}{\partial \tau}=-2 \beta P, \quad P=\int_{-\infty}^{\infty} A^{2} d X
\end{aligned}
$$

for mass and wave action flux, respectively. These express the conservation of $M, P$ in the conservative case when $\beta=0$, and also the conservation of $B, B^{2}$ in the transformed equation (7), respectively. 
Although the focus in this paper is on water waves, it is useful to describe here the general derivation for both surface and internal waves. The application to internal waves will follow in a subsequent paper. Thus, we assume that in the ocean, the background state varies slowly in the horizontal direction due to varying depth, and slow variations in the basic state hydrology and background currents. That is, the depth $h=h(x)$, the background horizontal current $u_{0}=u_{0}(z ; x)$ with a corresponding vertical velocity field $w_{0}(z, x)$, a density field $\rho_{0}(z ; x)$, a corresponding pressure field $p_{0}(z ; x)$, and a free surface displacement $\eta_{0}(x)$. This basic state satisfies the full steady-state Euler equations, with the exception of the momentum equations where there are body forces $F_{0}(z ; x), G_{0}(z ; x)$, respectively. That is,

$$
\begin{gathered}
\rho_{0}\left(u_{0} u_{0 x}+w_{0} u_{0 z}\right)+p_{0 x}=F_{0}, \\
{\left[\rho_{0}\left(u_{0} w_{0 x}+w_{0} w_{0 z}\right)\right]+p_{0 z}+g \rho_{0}=\left[G_{0}\right],} \\
u_{0} \rho_{0 x}+w_{0} \rho_{0 z}=0, \\
u_{0 x}+w_{0 z}=0, \\
w_{0}+u_{0} h_{x}=0 \quad \text { at } \quad z=-h(x), \\
p_{0}=0, \quad \text { at } \quad z=\eta_{0}, \\
u_{0} \eta_{0 x}=w_{0}, \quad \text { at } z=\eta_{0} .
\end{gathered}
$$

Because the $x$-dependence is slow, technically $\partial / \partial x \sim \epsilon^{3}, w_{0} \sim \epsilon^{3}, F_{0} \sim \epsilon^{3}$, and $G_{0} \sim \epsilon^{6}$, the dominant balance in the vertical momentum equation is hydrostatic and the terms in [.] can be omitted. This basic state is then perturbed by the wave field, where at the leading order the vertical particle displacement is given by

$$
\zeta \sim \eta(x, t) \phi(z ; x)
$$

Here, the modal function $\phi(z ; x)$ together with the linear long wave speed $c(x)$ is defined by the boundary-value problem

$$
\begin{gathered}
\left\{\rho_{0}\left(c-u_{0}\right)^{2} \phi_{z}\right\}_{z}-g \rho_{0 z} \phi=0, \quad \text { for } \quad-h<z<\eta_{0}, \\
\phi=0 \quad \text { at } \quad z=-h, \quad\left(c-u_{0}\right)^{2} \phi_{z}=g \phi \quad \text { at } \quad z=\eta_{0} .
\end{gathered}
$$


Continuation of this asymptotic expansion to the next order than yields the vKdV equation (1), see Grimshaw [5], Zhou and Grimshaw [6] or the reviews by Grimshaw [9], Grimshaw et al. [10]. The coefficients are given by

$$
\begin{gathered}
I \mu=3 \int_{-h}^{\eta_{0}} \rho_{0}\left(c-u_{0}\right)^{2} \phi_{z}^{3} d z, \\
I \delta=\int_{-h}^{\eta_{0}} \rho_{0}\left(c-u_{0}\right)^{2} \phi^{2} d z, \\
\text { where } \quad I=2 \int_{-h}^{\eta_{0}} \rho_{0}\left(c-u_{0}\right) \phi_{z}^{2} d z, \\
\text { and } Q=c^{2} I, \quad I \sigma=-\int_{-h}^{\eta_{0}} \phi \phi_{z} F_{0 z} d z .
\end{gathered}
$$

These expressions are well-known and much used, except for the coefficient $\sigma$ expressing nonconservative effects, whose derivation can also be obtained directly from the general theory for wave action presented in the review by Grimshaw [7].

As above, in this paper we focus only on the application of this general theory to water waves, where we assume that the background density is a constant, but in the presence of a background current. The application to internal waves will be presented in a subsequent paper. In Section 2, we first review the asymptotic theory for modulated periodic and solitary waves, emphasizing the effect of the hitherto neglected coefficient $\beta$. Then in Section 3, we examine the interaction of water waves with a background current, for the two cases when the background current has either no vertical shear, or a constant vertical shear. We conclude in Section 4.

\section{Modulated periodic and solitary waves}

When the coefficient $\alpha$ in (5) is a constant, and the coefficient $\beta=0$, the resulting $\mathrm{KdV}$ equation supports a periodic traveling wave, $A(X-V \tau)$, the well-known cnoidal wave solution

$$
\begin{gathered}
A=a\left\{b(m)+\operatorname{cn}^{2}(\gamma \theta ; m)\right\}+d, \quad \theta=k(X-V \tau), \\
\text { where } \alpha a=12 m \gamma^{2} k^{2} . \quad b(m)=\frac{1-m}{m}-\frac{E(m)}{m K(m)},
\end{gathered}
$$




$$
V-\alpha d=\frac{\alpha a}{3}\left\{\frac{2-m}{m}-\frac{3 E(m)}{m K(m)}\right\}=4 \gamma^{2} k^{2}\left\{2-m-\frac{3 E(m)}{K(m)}\right\} .
$$

Here, $\operatorname{cn}(x ; m)$ is the Jacobian elliptic function of modulus $m, 0<m<1$, and $K(\mathrm{~m})$ and $E(\mathrm{~m})$ are the elliptic integrals of the first and second kind. The expression (24) has period $2 \pi$ in $\theta$ so that $\gamma=K(m) / \pi$, while the spatial period is $2 \pi / k$. The (trough-to-crest) amplitude is $a$ and the mean value over one period is $d$. It is a three-parameter family with parameters $k, m, d$ say. As the modulus $m \rightarrow 1$, this becomes a solitary wave, since then $b \rightarrow 0$ and $\operatorname{cn}(x) \rightarrow \operatorname{sech}(x)$, while $\gamma \rightarrow \infty, k \rightarrow 0$ with $\gamma k=\Gamma$ fixed. As $m \rightarrow 0, b \rightarrow-1 / 2, \gamma \rightarrow 1 / 2, \operatorname{cn}(x) \rightarrow \cos (x)$, and it reduces to a sinusoidal wave $(a / 2) \cos (\theta)$ of small amplitude $a \sim m$ and wavenumber $k$.

We now allow this cnoidal wave to vary slowly with $\tau$; that is, the parameters $k, m, d$ vary slowly with $\tau$. The required modulation equations can be derived by an asymptotic expansion, or more directly from the available conservation laws, as in the original derivation by [11,12]. As three modulation equations are needed, and there are only two conservation laws available, we supplement $(8,9)$ with the equation for conservation of waves

$$
k_{\tau}+(k V)_{X}=0 .
$$

Since here we are allowing only $\tau$-modulations, it follows that $k$ is a constant. The remaining two modulation equations are obtained by inserting the cnoidal wave solution into the conservation laws $(8,9)$ and averaging over the phase $\theta$. The outcomes are

$$
\begin{gathered}
d_{\tau}=-\beta d, \\
\mathcal{P}_{\tau}=-2 \beta \mathcal{P}, \quad \mathcal{P}=<A^{2}>,
\end{gathered}
$$

where the $\langle\cdots\rangle$ denotes a $2 \pi$-average over $\theta$. The expression $\mathcal{P}$ is given by

$$
\begin{aligned}
\mathcal{P} & =d^{2}+a^{2}\left\{C_{4}-b^{2}\right\}, \\
C_{4} & =\frac{1}{3 m^{2} K(m)}\left\{3 m^{2} K(m)-5 m K(m)+4 m E(m)+2 K(m)-2 E(m)\right\} .
\end{aligned}
$$

Note that the equation for the mean level (28) is uncoupled from (29) and can be solved independently. Here, we choose $d=0$.

In the solitary wave limit $m \rightarrow 1$ and then $b \sim-1 / K(m)$ and $C_{4} \sim 2 / 3 K(m)$. The cnoidal wave expression (24), with $d=0$, becomes

$$
\left.A=a \operatorname{sech}^{2}(\Gamma \theta)\right\}, \quad \theta=X-V \tau, \quad V=\frac{\alpha a}{3}=12 \Gamma^{2}
$$


with one parameter to be determined. This is obtained from a reduction of (29) as $m \rightarrow 1$, or more directly by averaging the wave action conservation law (9) directly for a solitary wave, see Grimshaw [13] and the discussion in El et al. [14], Grimshaw and Yuan [8],

$$
\left\{\frac{a^{2}}{\Gamma}\right\}_{\tau}=-2 \beta\left\{\frac{a^{2}}{\Gamma}\right\} .
$$

Then, using the expressions in (31), this becomes

$$
\begin{gathered}
\mathcal{A}_{\tau}=-2 \beta \mathcal{A}, \quad \mathcal{A}=\left\{\frac{a^{3}}{\alpha}\right\}^{1 / 2}, \\
\frac{a^{3}}{a_{0}^{3}}=\frac{\alpha}{\alpha_{0}} \exp \left(-4 \int_{0}^{\tau} \beta d \tau\right),
\end{gathered}
$$

where the " 0 " subscript denotes the value at $\tau=0$. In terms of the original $\mathrm{vKdV}$ equation (1) the amplitude of the solitary wave is $a_{s}=a Q^{-1 / 2}$ and taking account of (4), (34) becomes

$$
\frac{a_{s}^{3}}{a_{s 0}^{3}}=\frac{\kappa}{\kappa_{0}} \exp \left(-4 \int_{0}^{T} \sigma d T\right), \quad \kappa=\frac{c^{2} \mu}{Q^{2} \delta} .
$$

The case of a periodic wave modulated only in $\tau$ was studied by Grimshaw $[9,15]$ and is reproduced briefly here. We recall that here $d=0$, and then, using the expressions in (25), (29) becomes

$$
\frac{F(m)}{F\left(m_{0}\right)}=\frac{\alpha^{2}}{\alpha_{0}^{2}} \exp \left(-2 \int_{0}^{\tau} \beta d \tau\right),
$$

where $\quad F(m)=K(m)^{2}\left\{(4-2 m) E(m) K(m)-3 E(m)^{2}-(1-m) K(m)^{2}\right\}$.

A plot of the normalized $F(m)$ (the left-hand side of (36)) when $m_{0}=0.98$ (a strongly nonlinear wave) and $m_{0}=0.85$ is shown in Fig. 1. We see that when $\beta=0$, then as $|\alpha|$ increases/decreases, so does the modulus $m$. Substitution into the expression (25) for the wave amplitude yields

$$
\frac{a}{a_{0}}=\frac{m K(m)^{2} \alpha_{0}}{m_{0} K\left(m_{0}\right)^{2} \alpha}
$$

This expresses the normalized amplitude in terms of the modulus $m$ and $\alpha$, and $m$ in turn varies with $\alpha$ according to (36). A plot of (38) when $\beta=0$ is shown in Fig. 2 when $m_{0}=0.98,0.85$, and shows that as $|\alpha|$ 


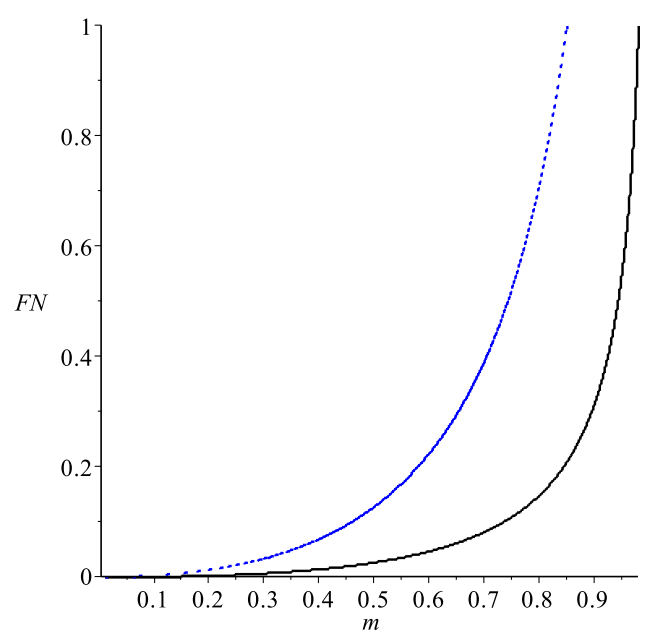

Figure 1. (color online) A plot of the normalized $F N=F(m) / F\left(m_{0}\right)$ (36) versus $m$ when $m_{0}=0.98$ (lower curve, black, solid) and $m_{0}=0.85$ (upper curve, blue, dash).

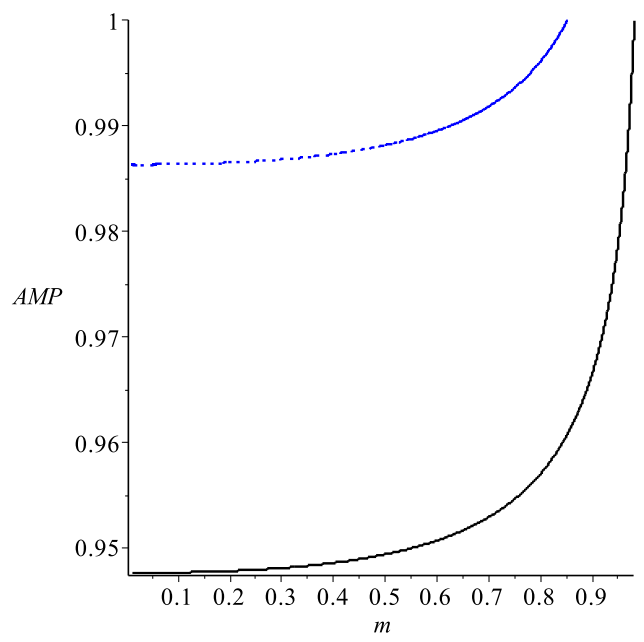

Figure 2. (color online) A plot of the normalized amplitude $A M P=a / a_{0}$ (38) versus $m$ when $m_{0}=0.98$ (lower curve, black, solid) and $m_{0}=0.85$ (upper curve, blue, dash).

increases/decreases then so does the amplitude. In both cases, there is a surprisingly small variation of the amplitude $a$ until $m \rightarrow 1$. As for the solitary wave, in terms of the original $\mathrm{KdV}$ equation (1), the amplitude of the periodic wave $a_{p}=a Q^{-1 / 2}$ and $\alpha=c^{2} \mu / Q^{1 / 2} \delta$. 


\section{Water waves on a background current}

\subsection{Current with zero background shear}

For this application, we put $\rho_{0}(z ; x)=\rho_{1}$, a constant, and assume that the basic current $u_{0}(z ; x)=U(x)$ does not depend on $z$. Then the basic state equations (10)-(16) reduce to

$$
\begin{gathered}
\rho_{1}\left(U U_{x}+g \eta_{0 x}\right)=F_{0}, \\
p_{0}=g \rho_{1}\left(\eta_{0}-z\right), \\
H U=M_{0}, \quad H=h+\eta_{0},
\end{gathered}
$$

where $M_{0}$ is the constant mass flux. Importantly, note that $F_{0}$ does not depend on $z$ and so $\sigma=0$ in this case, see (23). The modal function (18, $19)$ and the coefficients (20)-(23) are now given by

$$
\begin{gathered}
\phi \phi=\frac{z+h}{H}, \quad \text { for } \quad-h<z<\eta_{0}, \\
\text { where } \quad c=U+c_{0}, \quad c_{0}=(g H)^{1 / 2},
\end{gathered}
$$

$$
\text { and so } \quad \mu=\frac{3 c_{0}}{2 H}, \quad \delta=\frac{c_{0 H}^{2}}{6}, \quad I=\frac{2 c_{0}}{H}, \quad Q=\frac{2 g c^{2}}{c_{0}}, \quad \sigma=0 .
$$

Note that $\phi(z)$ is normalized so that $\phi\left(z=\eta_{0}\right)=1$ and then $\eta$ is the surface wave amplitude. We assume that $c>0$ and so the waves move in the positive $x$-direction.

For application to the slowly varying solitary wave, the expression (35) for the amplitude of a solitary wave reduces to

$$
\frac{a_{s}^{3}}{a_{s 0}^{3}}=\frac{\kappa}{\kappa_{0}}, \quad \kappa=\frac{9}{4 g c^{2} H^{2}} .
$$

Using the expression (41) this becomes

$$
\frac{a_{s}^{3}}{a_{s 0}^{3}}=\frac{\kappa}{\kappa_{0}}, \quad \kappa=\frac{9}{4 g\left\{g^{1 / 2} H^{3 / 2}+M_{0}\right\}^{2}} .
$$

When $U=0, M_{0}=0$ and this is the usual well-known law that $a_{s} \propto H^{-1}$. In an advancing current, $U>0, M_{0}>0, a_{s}$ increases as $U$ increases and $H$ decreases, but the rate of increase is reduced from that when $U=0$. On the 
other hand in an opposing current, $U<0, M_{0}<0, a_{s}$ again increases as $|U|$ increases and $H$ decreases, but now the rate of increase is enhanced, and $a_{s} \rightarrow \infty$ when $c \rightarrow 0, U \rightarrow-\left(g\left|M_{0}\right|\right)^{1 / 3}$. Note that this limit corresponds to critical flow, as then the Froude number $|U| / c_{0} \rightarrow 1$ and we infer that the breakdown corresponds to the failure of the quasi-steady hypothesis at criticality. However, note that this occurs as $c \rightarrow 0$ and then arises only as $T \rightarrow \infty$, see (2).

For a periodic wave, we use the expressions $(36,38)$ where

$$
\begin{gathered}
\alpha=\frac{9 c c_{0}^{1 / 2}}{(2 g)^{1 / 2} H^{3}}=\frac{9\left\{g^{1 / 2} H^{3 / 2}+M_{0}\right\}}{(4 g)^{1 / 4} H^{15 / 4}}, \\
a_{p}=\frac{a c_{0}^{1 / 2}}{(2 g)^{1 / 2} c}=\frac{a H^{5 / 4}}{(4 g)^{1 / 4}\left\{g^{1 / 2} H^{3 / 2}+M_{0}\right\}} .
\end{gathered}
$$

When $U=0, M_{0}=0$ and then $\alpha \propto H^{-9 / 4}$. Thus as the depth $H$ decreases, the modulus $m$ increases toward 1, the amplitude $a$ increases, and the wave develops into a solitary wave train. In this case, the amplitude $a_{p} \propto a H^{-1 / 4}$ and also increases. In an advancing current, $U>0, M_{0}>0$, the increase in $\alpha$ with decreasing depth $H$ is enhanced, and so again the modulus $m$ increases toward 1 and the amplitude $a$ increases. Indeed, as $m \rightarrow 1, F(m) \approx 2 K(m)^{3}$, see (37), and the expression (47) reduces to the solitary wave law (46). On the other hand, in an opposing current, $U<0, M_{0}<0$, the expression (47) shows that when $H^{3 / 2}>5\left|M_{0}\right| / 3 g^{1 / 2}$ and the depth $H$ decreases, $\alpha$ increases toward a maximum of $C_{1} g /|M|^{3 / 2}$, $C_{1}=(3 / 5)^{1 / 2} 27 / 25$, the modulus $m$ increases toward 1 , the solitary wave limit (46) may be reached and so the amplitude increases. However, when $H^{3 / 2}<5\left|M_{0}\right| / 3 g^{1 / 2}, \alpha$ decreases, the modulus $m$ decreases and indeed becomes 0 when $H^{3 / 2}=\left|M_{0}\right| / g^{1 / 2}$, that is $c=0$, although this limit is only reached as $\tau \rightarrow \infty$. In this regime, then $F(m) \sim m^{2}$ and the expression (38) shows that the amplitude $a$ tends to a constant. However, the physical amplitude $a_{p}$ increases and becomes infinite as $m \rightarrow 0$.

In Fig. 3, we show two typical numerical simulations of the vKdV equation (5) for cases when $U>0$ and $U<0$. The initial condition is the solitary wave (31) with nondimensional amplitude $a=0.1$ and nondimensional $\Gamma=0.14$. In these simulations, we use nondimensional coordinates such that $h=1, g=1$ and choose $U= \pm(0.1+0.2 \tanh (0.001 \tau)), M_{0}= \pm 0.1$, respectively, corresponding to an advancing or opposing current in $x>0$. Note that as $\tau \rightarrow \infty$. $H \rightarrow 1 / 3$. Then $\alpha$ is given by (47) using the relation (41); note that when $H=1, \alpha=7.00,5.73$, respectively, at the initial location $\tau=0$, and in both cases increases as $|U|$ increases and $H$ decreases, reaching $\alpha=107.1,35.6$ at $\tau=2000$. Thus, in the case $U>0$, $\alpha$ is much larger than for the case $U<0$, and consequently the solution for 

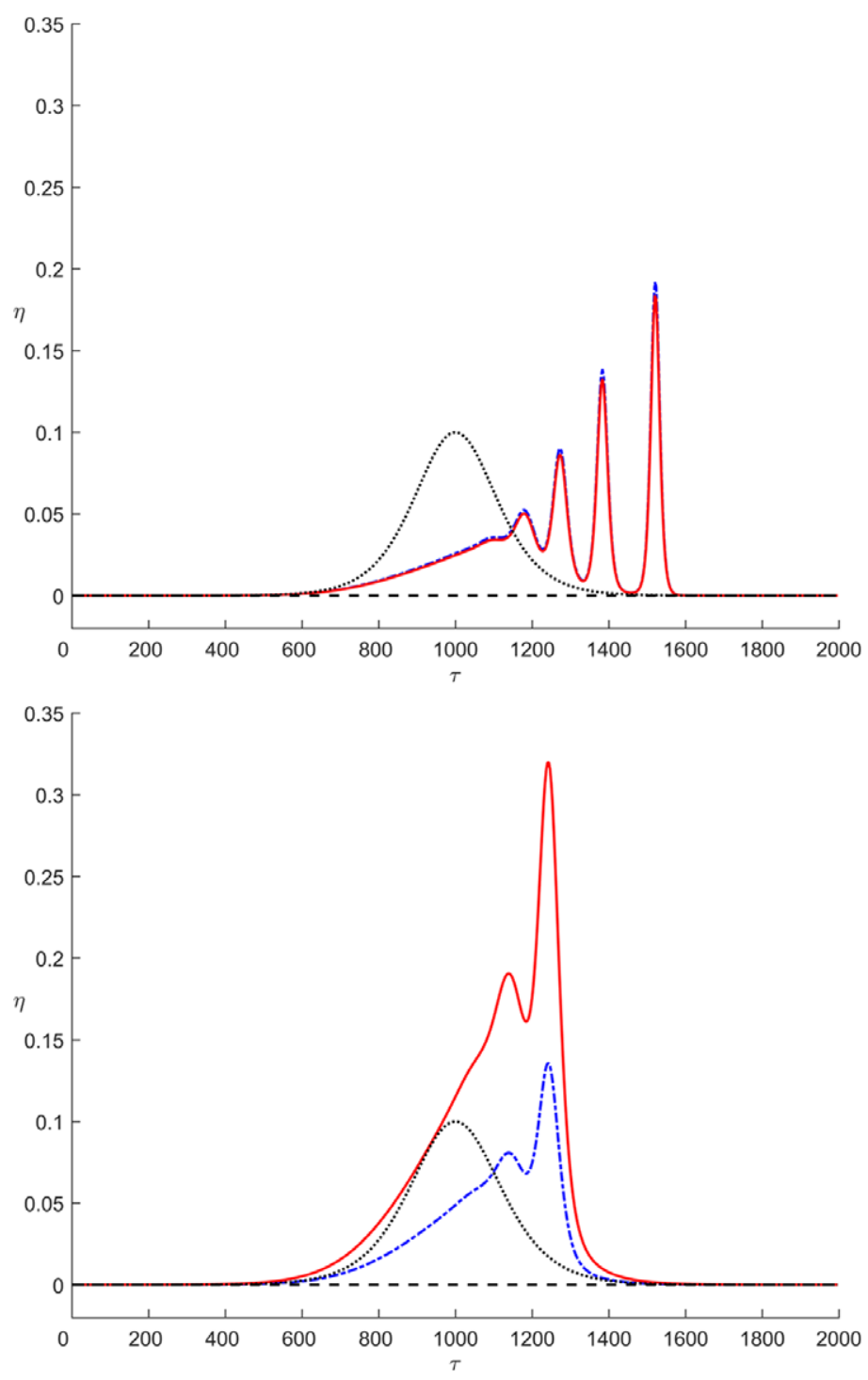

Figure 3. (color online) A numerical simulation of the $\mathrm{vKdV}$ equation (5) for cases when $U>0$ (top) and $U<0$ (bottom). The initial condition is the solitary wave (31) (black, dot), the numerical solution is $A$ (blue, dash), and the physical solution is $\eta$ (red, solid).

the transformed variable $A$ is larger and shows more fissioning when $U>0$ than when $U<0$ due to the enhanced nonlinearity. However, the solution magnitude for the physical variable $\eta$ is $\eta=A Q^{-1 / 2}$ (2) where here $Q$ is given in (44). In the case $U>0, Q$ varies very little, for $Q=1$ at $\tau=0$ to $Q=1.09$ at $\tau=2000$, and the decrease from $A$ to $\eta$ is less than $5 \%$. 
However, in the case $U<0, Q$ decreases from $Q=1$ at $\tau=0$ to $Q=0.2$ at $\tau=2000$ and consequently there is a substantial increase from $A$ to $\eta$. This is primarily due to the factor $c^{2}$ in $Q$, as here $c$ decreases toward zero as $|U|$ increases. However, note that this effect of the factor $\sqrt{Q}$ influences only the magnitude and does not change the fissioning dynamics.

\subsection{Linear shear}

For this application, we now again put $\rho_{0}(z ; x)=\rho_{1}$ is a constant, but assume that the basic current $u_{0}(z ; x)=U_{b}(x)+\Omega(x)(z+h)$ has a linear vertical shear, and $U_{b}(x)$ is the bottom velocity. Now the basic state equations (10)-(16) reduce to

$$
\begin{gathered}
\rho_{1}\left(U_{b} U_{b x}+U_{b} \Omega_{x}(z+h)+\Omega \Omega_{x} \frac{(z+h)^{2}}{2}+g \eta_{0 x}\right)=F_{0}, \\
p_{0}=g \rho_{1}\left(\eta_{0}-z\right), \\
U H=M_{0}, \quad H=h+\eta_{0}, \quad U=U_{b}+\frac{\Omega H}{2},
\end{gathered}
$$

where $U$ is the depth-averaged velocity and $M_{0}$ is again the constant mass flux. However, now $F_{0}$ depends on $z$

$$
F_{0 z}=\Omega_{x} u_{0},
$$

and so $\sigma(23)$ is not zero. Note here that a factor $\rho_{1}$ has been omitted. The modal function $(18,19)$ and the coefficients $(20)-(23)$ are now given by

$$
\begin{gathered}
\phi=\frac{\left(c-U_{b}-\Omega H\right)(z+h)}{\left(c-u_{0}\right) H}, \quad \text { for }-h<z<\eta_{0}, \\
\hat{c}=c-U, \quad \hat{c}=\left\{c_{0}^{2}+\Omega^{2} H^{2} / 4\right\}^{1 / 2}, \quad \phi_{z}=\frac{c_{0}^{2}}{H\left(c-u_{0}\right)^{2}}, \quad I=\frac{2 \hat{c}}{H}, \\
\mu=\frac{3 c_{0}^{2}+\Omega^{2} H^{2}}{2 H \hat{c}}, \quad \delta=\frac{(\hat{c}-\Omega H / 2)^{2} H^{2}}{6 \hat{c}}, \quad Q=\frac{2 g c^{2} \hat{c}}{c_{0}^{2}} \\
\sigma=\frac{\Omega_{x} H}{4 \hat{c}}(-U+\hat{c} F), \quad \beta=\frac{\Omega_{\tau} H}{4 c \hat{c}}(-U+\hat{c} F), \\
\text { where } \quad F=-1+\frac{\left(1-\omega^{2}\right)}{\omega}\left\{1+\frac{1-\omega}{2 \omega} \log \frac{(1-\omega)}{(1+\omega)}\right\}, \omega=\frac{\Omega H}{2 \hat{c}} .
\end{gathered}
$$




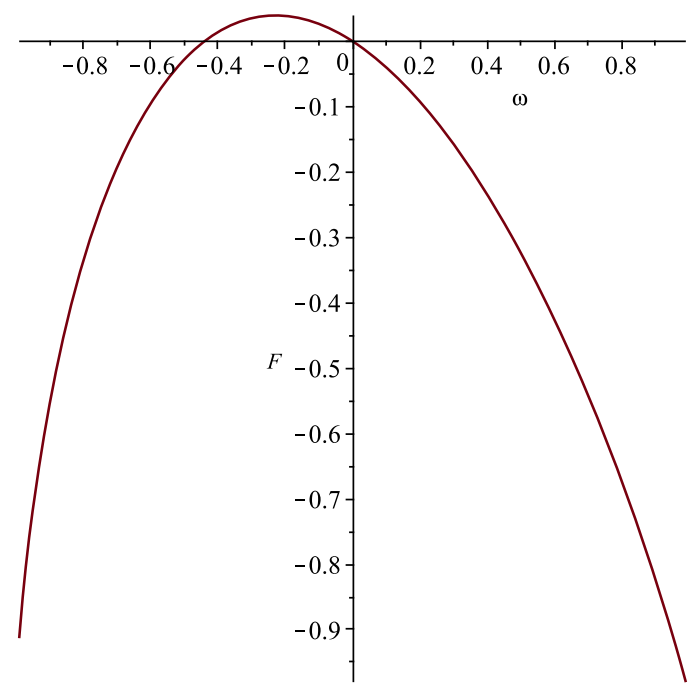

Figure 4. A plot of the $F(\omega)(55)$.

Here, $\phi(z)$ is again normalized so that $\phi\left(z=\eta_{0}\right)=1$ and so $\eta$ is the surface wave amplitude. We have chosen a plus sign in (53) so that $\hat{c}>0$. Note that $\mu, \delta, \hat{c}$ depend only on $\Omega, H$, and the speed $U$ enters explicitly only into $Q, \beta$. In particular, the expression $F$ in (55) depends only on the nondimensional shear $\omega$ where $|\omega|<1$. In the limit of weak shear when $\omega \rightarrow 0, F \rightarrow 0$ and so

$$
\beta \rightarrow-\frac{\Omega_{\tau} H U}{4 c c_{0}}, \quad \text { as } \quad \omega \rightarrow 0 .
$$

Thus, in this limit $\beta<0$ in an advancing current $(U>0)$ when the shear gradient is increasing $\left(\Omega_{\tau}>0\right)$, while $\beta<0$ in an opposing current $(U<0)$ when the shear gradient is decreasing $\left(\Omega_{\tau}<0\right)$; otherwise $\beta>0$. In general, a plot of $F(\omega)$ is shown in Fig. 4, and we see that $F$ varies between a minimum -1 at $\omega= \pm 1$ and a maximum of 0.03936 at $\omega=-0.2288$. Furthermore, $F>0$ only in the range $-0.4393<\omega<0$ and $F<0$ elsewhere. It follows that in advancing current $U>0$, the conclusion based on (56) that $\beta<0$ when $\Omega_{\tau}>0$ still holds for all $-1<\omega<-0.4393$ and $0<\omega<1$, and can be reversed only if $-0.4393<\omega<0$ and only then if also $U<0.03936 \hat{c}$. On the other hand in an opposing current $U<0$, the conclusion based on (56) that $\beta<0$ when $\Omega_{\tau}<0$ still holds only when $-0.4393<\omega<0$, but may be reversed if $-1<\omega<-0.4393$ or $0<\omega<1$ provided that also $|U|<\hat{c}$. 
For the application to the slowly varying solitary wave, the expression (35) reduces to

$$
\frac{a_{s}^{3}}{a_{s 0}^{3}}=\frac{\kappa}{\kappa_{0}} \exp \left(-4 \int_{0}^{x} \frac{\sigma d x}{c}\right), \quad \kappa=\frac{c^{2} \mu}{Q^{2} \delta}=\frac{3}{4 H c^{2} \hat{c}^{2}} \frac{\left(3 c_{0}^{2}+\Omega^{2} H^{2}\right)}{(\hat{c}-\Omega H / 2)^{2}}
$$

In the absence of a background shear $\left(\Omega=0, \hat{c}=c_{0}\right)$ this reduces to (46) and so the main interest now is the effect of including shear. Thus we examine the effect of $\Omega$ with $H, U$ fixed. In the adiabatic term $\kappa$ increasing $|\Omega|$ increases $\hat{c}$, according to the term $\Omega^{2} H^{2} / c_{0}^{2}(53)$, but this term is typically quite small, being $O\left(\omega^{2}\right)$. The main effect is due to the term $1 /(\hat{c}-\Omega H / 2)^{2}$ which increases as $\omega$ increases, and is greater or less than $1 / c_{0}$ according as $\omega>0$ or $\omega<0$. An additional effect of the shear is in the nonconservative term since now $\sigma \neq 0$ and there is amplitude growth or decay according as $\sigma<0$ or $\sigma>0$, where the dependence on $\omega$ has been discussed above.

For a periodic wave, we use the expressions $(36,38)$ where now

$$
\alpha==\frac{3 c}{H^{5 / 2}(2 \hat{c})^{1 / 2}} \frac{\left(3 c_{0}^{2}+\Omega^{2} H^{2}\right)}{(\hat{c}-\Omega H / 2)^{2}}, \quad a_{p}=\frac{a c_{0}}{c(2 g \hat{c})^{1 / 2}}
$$

in the absence of a background shear $\left(\Omega=0, \hat{c}=c_{0}\right)$ this reduces to (47). The effect of the shear when $U, H$ are fixed is quite small. The main effect on $\alpha$ is through the term $(\hat{c}-\Omega H / 2)^{2}$ which decreases as $\omega$ increases, and is less or greater than $c_{0}$ according as $\omega>0$ or $\omega<0$. There is now an additional effect of the shear in the nonconservative term and this is the same as that described above for a solitary wave.

In Figs. 5 and 6, we show some typical numerical simulations of the $\mathrm{vKdV}$ equation (5) for cases when $U>0, \Omega_{\tau}>0(<0)$, and $U<0, \Omega_{\tau}>0(<0)$. As for the case when $\Omega=0$ shown in Section 3.1, we use nondimensional coordinates such that $h=1, g=1$ and choose $U= \pm(0.1+0.2 \tanh (0.001 \tau)), M_{0}= \pm 0.1$, respectively, corresponding to an advancing or opposing current in $x>0$. The initial condition is again the solitary wave (31) with nondimensional amplitude $a=0.1$ and nondimensional $\Gamma=0.14$. Here, we also now choose $\Omega= \pm 0.2 \tanh (0.001 \tau)$ for each choice of $U$. Note that as $\tau \rightarrow \infty,|U| \rightarrow 0.3,|\Omega H| \rightarrow 0.0667$, and $|\omega| \rightarrow 0.058$, and so the approximation (56) is a good indication of the possible signs of $\beta$. Then $\alpha$ is given by (58) using the relation (50); note that since $\Omega=0$ at $\tau=0$ then $\alpha=7.00,5.73$ at the initial location $\tau=0$, according as $U>0(<0)$. The effect of $\Omega$ is rather small, and, as above for the case $\Omega=0, \alpha$ increases as $\tau$ increases. When $\tau=2000, \alpha=121,97,41,33$ according as $U>0, \Omega>0, U>0, \Omega<0$, $U<0, \Omega>0, U<0, \Omega<0$, respectively. When $\Omega_{\tau}>0(<0), \beta>0(<0)$ and in all cases $|\beta|$ is quite small, of order $10^{-4}$. However, the effect 

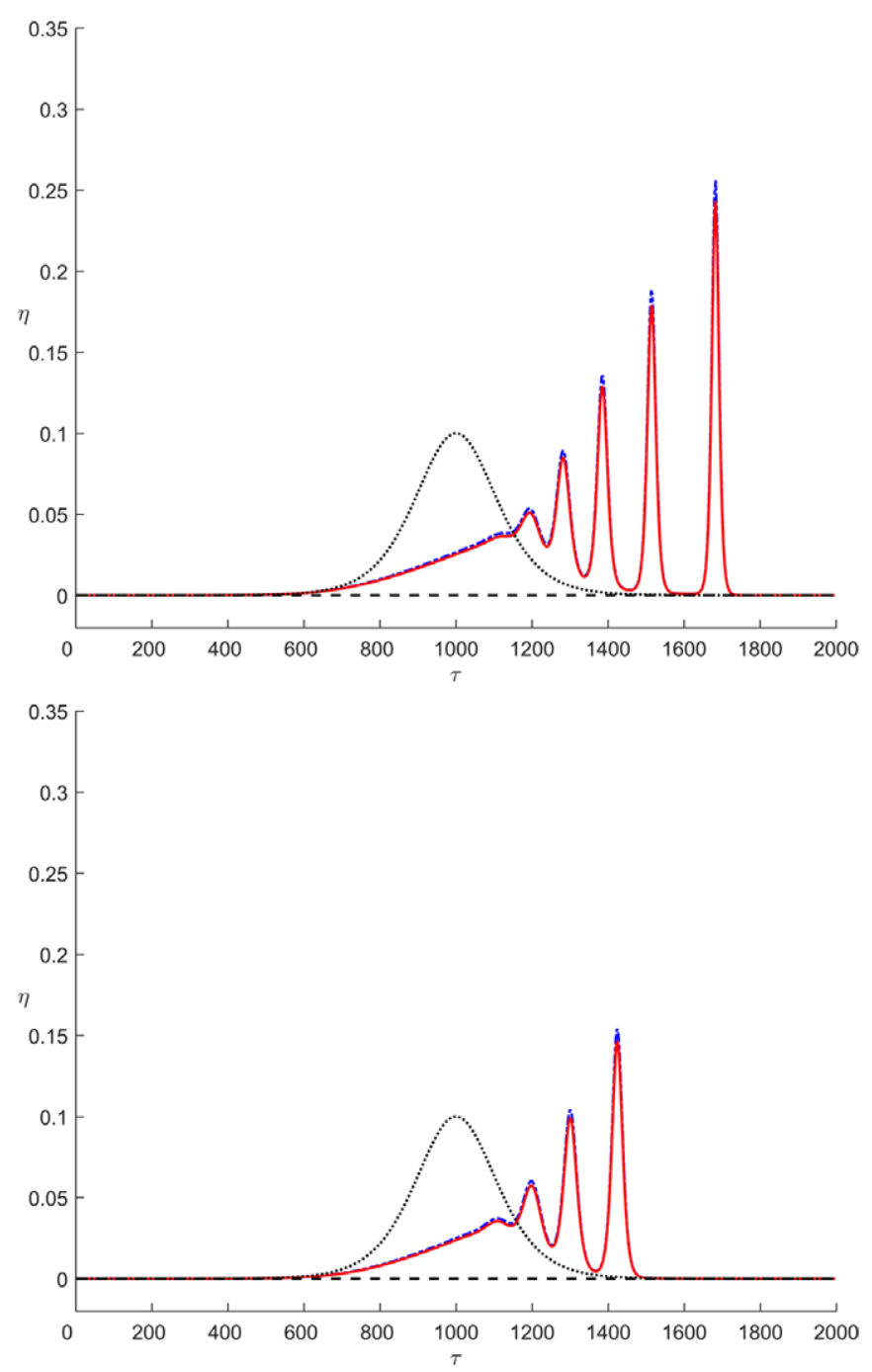

Figure 5. (color online) A numerical simulation of the $\mathrm{vKdV}$ equation (5) for cases when $U>0, \Omega_{\tau}>0$ (top) and $\Omega_{\tau}<0$ (bottom). The initial condition is the solitary wave (31) (black, dot), the numerical solution is $A$ (blue, dash) and the physical solution is $\eta$ (red, solid).

of $\beta$ is cumulative and of more relevance is $E$ which increases (decreases) from 1 at $\tau=0$ to $1.25,0.84,0.973,1.026$ at $\tau=2000$, according as $U>0, \Omega>0, U>0, \Omega<0, U<0, \Omega>0, U<0, \Omega<0$, respectively. Note that the effect of the $\beta$ term, although small, is much larger when $U>0$ than when $U<0$. The magnification factor $Q$ correspondingly varies from 1 at $\tau=0$ to $1.105,1.105,0.19,0.19$ (note that $Q$ depends only on $\Omega$ and is independent of the sign of $\Omega$ ), and as in the case when $\Omega=0$ 

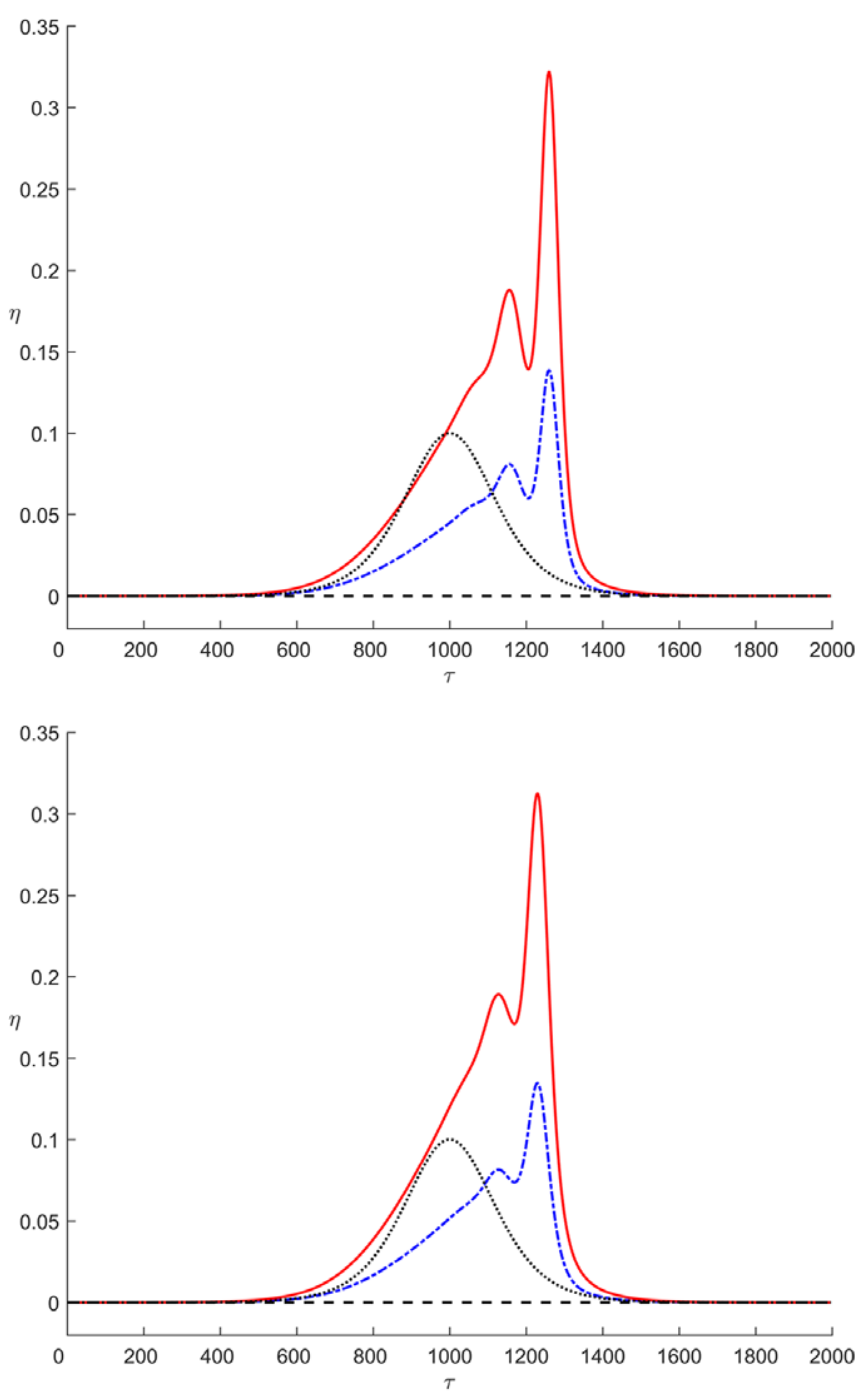

Figure 6. (color online) A numerical simulation of the $\mathrm{vKdV}$ equation (5) for cases when $U<0, \Omega_{\tau}>0$ (top), and $\Omega_{\tau}<0$ (bottom). The initial condition is the solitary wave (31) (black, dot), the numerical solution is $A$ (blue, dash), and the physical solution is $\eta$ (red, solid).

is ineffective when $U>0$, but very significant when $U<0$. Overall then the simulations are similar to those when $\Omega=0$. The dynamical fissioning is more apparent when $U>0$ due to the larger values of $\alpha$, but the overall amplitude growth is greater when $U<0$ due to the great decrease then in $Q$. The dynamical effect of the shear is overall rather small, but more significant when $U>0$ than when $U<0$. 


\section{Discussion}

In this paper, we have used the $\mathrm{vKdV}$ equation (5) with both numerical simulations and some theory to analyze the effect of a background current on the propagation of nonlinear periodic and solitary water waves. The motivation is to contrast this shallow water scenario with the much more studied case of deep water waves propagating on currents. Although in both cases conservation of waves and wave action play an essential role, and in both cases there is more wave amplification for waves on an opposing current than for waves on an advancing current, there are some major differences.

First, in this long wave limit, it is necessary to take account of both nonlinearity and wave dispersion and hence we have used a $\mathrm{KdV}$ model, developed for both surface and internal waves, and applied here only for water waves. A companion paper will examine the case of internal waves. Second, the horizontal variation in the background current is supported by a corresponding change in total depth, in order that total mass is conserved, and this depth change is the main element in the transformation of the waves as they propagate on the variable current. Third, when the background current has vertical shear, some body force is needed to maintain that shear, and this leads to a term in the $\mathrm{vKdV}$ equation (1) with coefficient $\sigma$ representing nonconservation of wave action; the analogous term in the transformed equation (5) has coefficient $\beta$. Although it transpires that this term is quite small for the numerical examples we have considered, it is nonetheless an important and hitherto neglected aspect of waves riding on currents, and this issue will be further developed when we examine the case of internal waves.

We have examined two typical case. In Section 3.1, the background current has no vertical shear, and consequently the aforementioned nonconservative term is absent, that is $\beta=0$. The outcome is that in both cases of an advancing or opposing current, there is wave amplification, due to the corresponding decrease in the total depth of fluid, but the increase is much more marked for the case of an opposing current, see Fig. 3. An important feature here is that dynamical nonlinearity is more important for the case of an advancing current, due to the larger value of the nonlinear coefficient $\alpha$ in the transformed equation (5) and this leads to greater fissioning of the amplitude $A$ in that case. However, the transformation back to the physical variable $\eta$ through the transformation $\eta=A Q^{-1 / 2}$ (2) makes very little difference for the case of an advancing current, but a substantial increase in amplitude for the case of an opposing current as $Q$ decreases as $|U|$ increases, due mainly to the decrease then in the speed $c$.

In Section 3.2, the background current has uniform background shear $\Omega$. The main consequence is that then $\beta \neq 0$ and for realistic values of $\Omega$ when the approximation (56) can be used, $\beta<0$ implying wave growth when $U, \Omega_{\tau}$ have the same sign, but $\beta>0$ implying wave decay when $U, \Omega_{\tau}$ have 
the opposite sign. However, for realistic values of $\Omega$ this nonconservative effect is not very large, and the amplitudes evolve on the current in a similar manner to that described in Section 3.1, see Figs. 5 and 6.

\section{Acknowledgments}

RG was supported by the Leverhulme Trust through the award of a Leverhulme Emeritus Fellowship.

\section{References}

1. O. M. PhilliPs, The Dynamics of the Upper Ocean, Cambridge University Press, Cambridge, 1969.

2. D. H. Peregrine, Interaction of water waves and currents, Adv. Appl. Mech. 16:9-117 (1976).

3. R. Smith, Giant waves, J. Fluid Mech. 77:417-431 (1976).

4. C. Kharif, E. Pelinovsky, and A. Slunyaev, Rogue Waves in the Ocean, Springer-Verlag, Berlin Heidelberg, 2009.

5. R. Grimshaw, Evolution equations for long nonlinear internal waves in stratified shear flows, Stud. Appl. Math. 65:159-188 (1981).

6. X. ZHOU and R. GRIMSHAW, The effect of variable currents on internal solitary waves, Dyn. Atmos. Oceans 14:17-39 (1989).

7. R. GRIMSHAw, Wave action and wave-mean flow interaction, with application to stratified shear flows, Ann. Rev. Fluid Mech. 16:11-44 (1984).

8. R. Grimshaw and C. Yuan, The propagation of internal undular bores over variable topography, Physica D 333:200-207 (2016).

9. R. Grimshaw, Internal solitary waves in a variable medium, Gesells. Angew. Math. 30:96-109 (2007).

10. R. Grimshaw, E. Pelinovsky, T. Talipova, and A. Kurkina, Internal solitary waves: Propagation, deformation and disintegration, Nonlinear Process. Geophys. 17:633-649 (2010).

11. G. B. Whitham, Non-linear dispersive waves, Proc. R. Soc. Lond. A 283:238-261 (1965).

12. G. B. Whitham, Linear and Nonlinear Waves, J. Wiley and Sons, New York, 1974.

13. R. Grimshaw, Slowly varying solitary waves. I. Korteweg-de Vries equation, Proc. R. Soc. 368A:359-375 (1979).

14. G. El, R. Grimshaw, and W. Tiong, Transformation of a shoaling undular bore, J. Fluid Mech. 709:371-395 (2012).

15. R. GRIMSHAW, Change of polarity for periodic waves in the variable-coefficient Korteweg-de Vries equation, Stud. Appl. Math. 134:363-371 (2015).

UNIVERSITY COLLEGE LONDON

(Received October 26, 2016) 